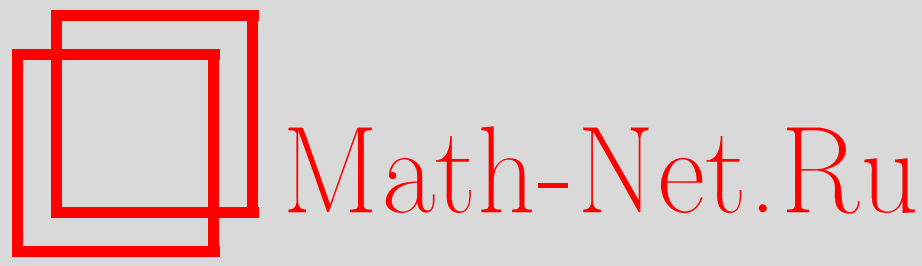

Ф. Бонеки, Ц. Цю, М. Тарлини, Бигамильтонова система на грассманиане, TMФ, 2016, том 189, номер 1, 3-14

DOI: https://doi.org/10.4213/tmf9087

Использование Общероссийского математического портала Math-Net.Ru подразумевает, что вы прочитали и согласны с пользовательским соглашением http://www . mathnet.ru/rus/agreement

Параметры загрузки:

IP : 3.85 .5 .30

26 апреля 2023 г., $04: 16: 09$

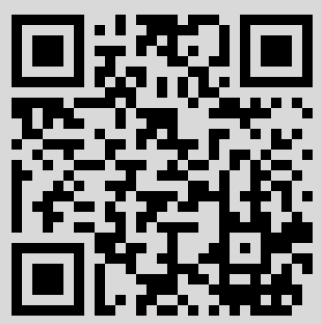




\title{
БИГАМИЛЬТОНОВА СИСТЕМА НА ГРАССМАНИАНЕ
}

\begin{abstract}
С учетом недавних результатов о том, что геометрия Пуассона-Нийенхейса соответствует квантованию симплектического группоида, интегрирующего пуассоново многообразие, обсуждается структура Пуассона-Нийенхейса на грассманиане, заданном совместными структурами Кириллова--Костанта--Сурьо и БрюаПуассона. Собственные числа тензора Нийенхейса являются переменными Гельфанда-Цетлина, которые, как было показано, также находятся в инволюции по отношению к структуре Брюа-Пуассона. Кроме того, доказано, что расслоение Штифеля на грассманиане допускает наличие бигамильтоновой структуры.
\end{abstract}

Ключевые слова: симплектическая геометрия, интегрируемые системы, геометрия Пуассона-Нийенхейса, квантование пуассоновых многообразий, симплектические группоиды.

DOI: $10.4213 / \operatorname{tmf} 9087$

\section{1. ВВЕДЕНИЕ}

Понятие симплектического группоида было введено Карасевым и Вейнштейном с целью квантования пуассоновых многообразий. Действительно, если пуассоново многообразие $(M, \pi)$ интегрируемо, то существует каноническое симплектическое многообразие $\mathcal{G}$, наделенное совместной группоидной структурой, которая воспроизводит тензор Пуассона $\pi$ на его пространстве единиц $M$. Совместность симплектической структуры и группоида определяется требованием, чтобы граф умножения был лагранжевым подмногообразием многообразия $\overline{\mathcal{G}} \times \overline{\mathcal{G}} \times \mathcal{G}$, где $\overline{\mathcal{G}}$ имеет противоположную симплектическую форму. Если $\mathcal{H}$ - пространство состояний, полученных с помощью некоторого квантования многообразия $\mathcal{G}$, то граф умножения квантуется вектором в пространстве $\mathcal{H}^{*} \otimes \mathcal{H}^{*} \otimes \mathcal{H}$, который можно понимать как отображение $m: \mathcal{H} \otimes \mathcal{H} \rightarrow \mathcal{H}$, задающее алгебраическую структуру на $\mathcal{H}$, которую следует считать алгеброй наблюдаемых, квантующей алгебру Пуассона на $M$.

В работе [1] была предложена общая концепция, основанная на геометрическом квантовании симплектического группоида, где результатом квантования является

*National Institute of Nuclear Physics, Sezione di Firenze, Firenze, Italy.

Email: francesco.bonechi@fi.infn.it, marco.tarlini@fi.infn.it

${ }^{\dagger}$ Uppsala University, Department of Mathematics, Uppsala, Sweden.

Email: jian.qiu@math.uu.se 
алгебра $C^{*}$. Идея заключается в поиске мультипликативной поляризации, совместной с группоидной структурой, которая позволяет построить свертку на пространстве поляризованных сечений.

Наша точка зрения и основное наблюдение состоят в том, что именно эта перспектива (а не наложение дополнительных условий на сложную задачу поиска подходящих поляризаций) позволяет найти больше сингулярных поляризаций, а именно сингулярные действительные поляризации.

В работах [2], [3] мы начали изучение квантования интегрируемых многообразий Пуассона с помощью сингулярных лагранжевых поляризаций симплектического группоида. Начав с проективной плоскости $\mathbb{C} P_{1}$, мы перешли к структуре БрюаПуассона на комплексных проективных плоскостях $\mathbb{C} P_{n}$. А именно, рассматривалась действительная поляризация, индуцирующая в пространстве лагранжевых листов структуру топологического группоида. В статье [3] введено понятие мультипликативной интегрируемости модулярной функции. Модулярная функция должна быть интегрируемой в обычном смысле, гамильтонианы в инволюции должны быть совместны с группоидной структурой таким образом, чтобы слои уровней наследовали структуру топологического группоида. Тогда можно рассмотреть подгруппоид листов Зоммерфельда и (при условии, что можно ввести систему Хаара) считать квантованием алгебру конволюций.

Источником таких интегрируемых систем является бигамильтонова геометрия. Действительно, как было показано, каждая невырожденная симплектическая структура Пуассона-Нийенхейса (ПН) порождает мультипликативную интегрируемую модель на симплектическом группоиде [4]. Именно эта структура положена в основу построений работы [3] для проективных плоскостей $\mathbb{C} P_{n}$.

Как показано в работе [5], симплектическая структура ПН существует для всех компактных эрмитовых симплектических пространств: действительно, форма, обратная к симплектической форме Кириллова--Константа--Сурьо (KKC) $\Omega_{\mathrm{kks}}$, и структура Брюа-Пуассона $\pi_{\text {bp }}$ совместны. В статье [6] доказано, что эти структуры ПН имеют максимальный ранг и, следовательно, задают мультипликативную интегрируемую модель на симплектическом группоиде, интегрирующем структуру БрюаПуассона. Доказательство является общим для всех классических случаев; в настоящей работе развивается более явный альтернативный подход, применимый в случае грассманианов. Мы предполагаем, что он окажется полезным при изучении мультипликативных интегрируемых моделей на симплектических группоидах. Кроме того, мы используем геометрическую концепцию, которая была использована в работе [7] при изучении вырождения торов системы Гельфанда-Цетлина, и, таким образом, представляется разумным попытаться найти возможное вырождение полной геометрии Нийенхейса.

В статье [6] в явном виде посчитаны собственные числа оператора Нийенхейса $N=\pi_{\mathrm{bp}} \Omega_{\mathrm{kks}}$ и доказано, что они представляют собой общеизвестные переменные Гельфанда-Цетлина. Эта интегрируемая модель хорошо известна, она изучена в работе [8]. Новым является тот факт, что собственные числа находятся в инволюции и со структурой Брюа-Пуассона. В данной работе мы начинаем с построения в явном виде структуры Пуассона на $\mathbb{C}^{n k}$ и сводим ее к грассманиану, следуя тем же этапам симплектической редукции, которая задает и симплектическую форму ККС, и доказываем далее более простым способом результаты, полученные в работе [6]. 
В частности, мы рассматриваем геометрию Пуассона расслоения Штифеля на грассманианах и, кроме того, доказываем существование бигамильтоновой структуры на многообразии Штифеля.

\section{2. СТРУКТУРЫ ПН}

Напомним основные необходимые факты из геометрии ПН. Пусть $(M, \pi)$ - пуассоново многообразие, где $\pi \in C^{\infty}\left(\Lambda^{2} T M\right)$ - бивектор Пуассона, и

$$
\{f, g\}=\pi^{i j} \partial_{i} f \partial_{j} g
$$

есть скобка Пуассона между $f, g \in C^{\infty}(M)$. С помощью скобки Схоутена между мультивекторными полями можно записать тождество Якоби для скобки Пуассона в виде $[\pi, \pi]=0$.

$(1,1)$-тензор $N: T M \rightarrow T M$ называется тензором Нийенхейса, если он имеет нулевое кручение Нийенхейса, т. е. для любой пары $\left(v_{1}, v_{2}\right)$ векторных полей на $M$ имеем

$$
T(N)\left(v_{1}, v_{2}\right)=\left[N v_{1}, N v_{2}\right]-N\left(\left[N v_{1}, v_{2}\right]+\left[v_{1}, N v_{2}\right]-N\left[v_{1}, v_{2}\right]\right)=0 .
$$

Многообразием Пуассона-Нийенхейса является триада $(M, \pi, N)$, где $(M, \pi)-$ пуассоново многообразие, а $N$ - тензор Нийенхейса, при этом $\pi$ и $N$ совместны, т. e.

$$
N \circ \pi=\pi \circ N^{*}, \quad\{\alpha, \beta\}_{N \pi}=\left\{N^{*} \alpha, \beta\right\}_{\pi}+\left\{\alpha, N^{*} \beta\right\}_{\pi}-N^{*}\{\alpha, \beta\}_{\pi},
$$

где

$$
\{\alpha, \beta\}_{\pi}=L_{\pi(\alpha)} \beta-L_{\pi(\beta)} \alpha-d\langle\pi, \alpha \wedge \beta\rangle
$$

при $\alpha, \beta \in \Omega^{1}(M)$, а $N^{*}$ означает дуальное отображение. Одним из важных свойств геометрии ПН является существование иерархии совместных пуассоновых структур $N^{k}(\pi)$, т. е. $\left[N^{k}(\pi), N^{s}(\pi)\right]=0$ для всех $k, s \geqslant 0$.

Рассмотрим теперь обратную симплектическую форму $\Omega^{-1}$ и другую совместную пуассонову структуру $\pi_{0}$, т. е. $\left[\Omega^{-1}, \pi_{0}\right]=0$. Как следствие существует пучок структур Пуассона $\pi_{t}=\pi_{0}+t \Omega^{-1}$ при $t \in \mathbb{R}$. Кроме того, $\left(M, \Omega^{-1}, N=\pi_{0} \Omega\right)$ является (симплектической) структурой ПН (доказательство см. в статье [9]).

Структуры ПН тесно связаны с интегрируемыми системами (общие ссылки на бигамильтоновы системы даны в работе [10]). Рассмотрим спектралъную проблему, связанную с симплектической структурой ПН, а именно задачу на собственные числа тензора $N$. Поскольку $N^{*}-\lambda=\Omega \circ\left(\pi_{0}-\lambda \Omega^{-1}\right)$ и $\pi_{0}-\lambda \Omega^{-1}$ является антисимметричным оператором, собственные числа тензора $N^{*}$ дважды вырождены, и число различных собственных чисел не превосходит $(\operatorname{dim} M) / 2$. Будем говорить, что тензор $N$ имеет максимальный ранг, если существует открытое плотное многообразие $M_{0}$, на котором определено $(\operatorname{dim} M) / 2$ независимых функций $\lambda_{i} \in C^{1}\left(M_{0}\right)$ таких, что $\lambda_{i}(x)$ является собственным числом тензора $N$ при $x \in M_{0}$. Назовем такие функции $\lambda_{i}$ собственными числами Нийенхейса. Нет гарантии, что их можно продолжить на все многообразие $M$; в наших примерах они продолжаются как непрерывные функции.

Набор гладких функций $I_{k}$ удовлетворяет рекуррентным соотношениям Ленарда, если

$$
d I_{k+1}=N^{*} d I_{k}
$$


Как следствие $I_{k}$ находятся в инволюции с $\Omega^{-1}$ и $\pi_{0}$. Канонический набор таких функций имеет вид $I_{k}=1 / k \operatorname{Tr} N^{k}, k=1, \ldots, n$. Можно проверить уравнение (1) с помощью соотношений $\mathcal{L}_{N X} N=N \mathcal{L}_{X} N$ при $X \in T M$.

Если $N$ имеет максимальный ранг, собственные числа Нийенхейса удовлетворяют следующему уравнению:

$$
N^{*} d \lambda_{i}=\lambda_{i} d \lambda_{i}
$$

Собственные числа Нийенхейса находятся в инволюции с обеими пуассоновыми структурами $\Omega^{-1}$ и $\pi_{0}$, они представляют собой возможный выбор переменных действия интегрируемой системы.

\section{3. БИГАМИЛЬТОНОВЫ СИСТЕМЫ НА ГРАССМАНИАНЕ}

В этом разделе вводятся симплектические и пуассоновы структуры на грассманиане. Грассманиан $\operatorname{Gr}(n, k)$ можно понимать как коприсоединенную орбиту группы $U(n)$, таким образом фиксируется симплектическая структура KKС. Наделив $U(n)$ стандартной структурой Пуассона-Ли, можно показать, что

$$
\operatorname{Gr}(n, k)=U(n) / U(n-k) \times U(k)
$$

является однородным пространством Пуассона; назовем эту структуру структурой Брюа-Пуассона. В работе [5] показана совместность двух указанных пуассоновых структур.

Дадим явное описание этих структур на $\operatorname{Gr}(n, k)$. В п. 3.1 грассманиан $\operatorname{Gr}(n, k)$ охарактеризован как симплектическая редукция пространства $\mathbb{C}^{n k}$, рассматриваемая как гамильтоново пространство $U(k)$. Это построение определяет $U(k)$-расслоение Штифеля на грассманиане. В п. 3.2 мы показываем, что та же редукция задает структуру Брюа-Пуассона на $\operatorname{Gr}(n, k)$. Наконец, в п. 3.3 обсуждается совместность в рамках указанной геометрической концепции. В частности, доказано существование бигамильтоновой структуры не только на $\operatorname{Gr}(n, k)$, но и на многообразии Штифеля.

3.1. Расслоение Штифеля. Отождествим $\mathfrak{u}(n)$ с антиэрмитовыми матрицами; дуальные матрицы $\mathfrak{u}(n)^{*}$ отождествим с пространством $\operatorname{Herm}(n)$ эрмитовых матриц со спариванием $\langle X, \xi\rangle=\operatorname{Im} \operatorname{Tr}(X \xi)$, где $X \in \mathfrak{u}(n)$ и $\xi \in \operatorname{Herm}(n)$. Таким образом, коприсоединенное действие отображается в присоединенное. Определим $\operatorname{Gr}(n, k)$ как присоединенную $U(n)$-орбиту подпространства $\rho \subset \operatorname{Herm}(n)$, где

$$
\rho=\operatorname{diag}(\underbrace{0, \ldots, 0}_{n-k}, \underbrace{1, \ldots, 1}_{k}) .
$$

Обозначим через $\Omega_{\mathrm{kks}}$ соответствующую симплектическую структуру $\mathrm{KKC}$, а через $\{\cdot, \cdot\}_{\mathrm{kks}}-$ соответствующую скобку Пуассона. Поскольку подгруппой изотропий для $\rho$ является $U(n-k) \times U(k)$, где $U(n-k)$ - минор порядка $n-k$ в левом верхнем углу и $U(k)$ - минор порядка $k$ в правом нижнем углу, отображение $\mu_{(n, k)}: U(n) \rightarrow$ $\operatorname{Gr}(n, k)$, заданное формулой

$$
\mu_{(n, k)}(g)=g \rho g^{-1}, \quad g \in U(n),
$$


отождествляет $\operatorname{Gr}(n, k)$ с однородным пространством $U(n) / U(n-k) \times U(k)$. Обозначим тем же символом $\mu_{(n, k)}$ отображение моментов для гамильтонова присоединенного $U(n)$-действия, причем $\mu_{(n, k)}(X)=\left\langle X, \mu_{(n, k)}\right\rangle$. Отображение эквивариантно, т. е. для любого $X, Y \in \mathfrak{u}(n)$ имеем

$$
\left\{\mu_{(n, k)}(X), \mu_{(n, k)}(Y)\right\}_{\mathrm{kks}}=\mu_{(n, k)}([X, Y]) .
$$

Переменными Гельфанда-Цетлина являются собственные числа $\lambda_{\alpha}^{(r)}, \alpha=1, \ldots, r$, минора порядка $r$ в левом верхнем углу отображения $\mu_{(n, k)}$ (см. статью [8]). Они удовлетворяют следующим неравенствам, которые задают так называемый конус Гельфанда-Цетлина $\mathcal{C}_{\mathrm{GC}}$

$$
\lambda_{\alpha}^{(n-i)} \leqslant \lambda_{\alpha}^{(n-i-1)} \leqslant \lambda_{\alpha+1}^{(n-i)}
$$

при $i=0, \ldots, n-1$ и $\lambda_{\alpha}^{(n)}=(\underbrace{0, \ldots, 0}_{n-k}, \underbrace{1, \ldots, 1}_{k})$. В качестве примера приведем схему грассманиана $\operatorname{Gr}(4,2)$ :

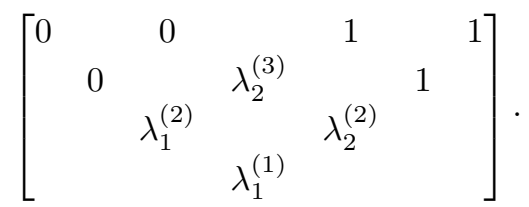

Грассманиан $\operatorname{Gr}(n, k)$ задается как симплектическая редукция пространства $\mathbb{C}^{n k}$. Рассмотрим каноническую симплектическую форму на $\mathbb{C}^{n k}$, записанную в виде

$$
\Omega=i \sum_{i, j} d z_{i}^{j} \wedge \bar{d} z_{i}^{j}, \quad i=1, \ldots, n, \quad j=n-k+1, \ldots, n .
$$

При заданном естественном правом $U(k)$-действии на векторном пространстве $\mathbb{C}^{n k}$ отображение его моментов имеет вид $\nu_{U(k)}: \mathbb{C}^{n k} \rightarrow \operatorname{Herm}(k)$, а именно:

$$
\nu_{U(k)}:\left(\begin{array}{ccc}
z_{1}^{n-k+1} & \ldots & z_{1}^{n} \\
\vdots & \ddots & \vdots \\
z_{n}^{n-k+1} & \ldots & z_{n}^{n}
\end{array}\right) \mapsto \sum_{i=1}^{n}\left(\begin{array}{ccc}
\left|z_{i}^{n-k+1}\right|^{2} & \ldots & \bar{z}_{i}^{n-k+1} z_{i}^{n} \\
\vdots & \ddots & \vdots \\
\bar{z}_{i}^{n} z_{i}^{n-k+1} & \ldots & \left|z_{i}^{n}\right|^{2}
\end{array}\right),
$$

при этом

$$
\sum_{j^{\prime}} z_{i}^{j^{\prime}} X_{j^{\prime} j}=\left\{\nu_{U(k)}(X), z_{i}^{j}\right\}
$$

где $z_{i}^{j}$ - координаты на $\mathbb{C}^{n k}$, а $X \in \mathfrak{u}(k)$.

Тогда многообразие

$$
\nu_{U(k)}^{-1}\left(1_{k}\right) \subset \mathbb{C}^{n k}
$$

характеризуется соотношениями

$$
\sum_{i=1}^{n}\left|z_{i}^{j}\right|^{2}=1, \quad \sum_{i=1}^{n} \bar{z}_{i}^{j} z_{i}^{j^{\prime}}=0, \quad j \neq j^{\prime}, \quad j, j^{\prime}=n-k+1, \ldots, n .
$$


С учетом этих выражений столбцы в правой части (7) образуют ортонормированный $k$-репер.

Получаем, что форма $\mathrm{KKC} \Omega_{\mathrm{kks}}$ на грассманиане $\operatorname{Gr}(n, k)$ является симплектической редукцией пространства $\mathbb{C}^{n k}$ под влиянием $U(k)$-действия:

$$
\operatorname{Gr}(n, k)=\mathbb{C}^{n k} / / U(k)=\nu_{U(k)}^{-1}\left(1_{k}\right) / U(k) .
$$

Подпространство пространства $\mathbb{C}^{n k}$, заданное с помощью $\nu_{U(k)}^{-1}\left(1_{k}\right)$, является многообразием Штифеля $S(n, k)$, а правое $U(k)$-действие является главным и задает главное расслоение Штифеля:

$$
\begin{aligned}
& S(n, k) \leftarrow U(k) \\
& \downarrow \\
& \operatorname{Gr}(n, k)
\end{aligned} .
$$

Это расслоение эквивариантно по отношению к естественным левым $U(n)$-действиям.

Кольцо полиномиальных функций $\mathcal{A}(S(n, k))$ образовано элементами матрицы $\sigma \in M_{n, k}(\mathcal{A}(S(n, k))$ вида

$$
\sigma=\left(\begin{array}{cccc}
z_{1}^{n-k+1} & z_{1}^{n-k+2} & \ldots & z_{1}^{n} \\
z_{2}^{n-k+1} & z_{2}^{n-k+2} & \ldots & z_{2}^{n} \\
\vdots & \ddots & & \vdots \\
z_{n}^{n-k+1} & z_{n}^{n-k+2} & \ldots & z_{n}^{n}
\end{array}\right)
$$

Тогда легко проверить, что

$$
\nu_{U(k)}=\sigma^{\dagger} \sigma=1_{k}, \quad \mu_{(n, k)}=\sigma \sigma^{\dagger},
$$

где $\mu_{(n, k)}$ - отображение моментов для гамильтонова левого $U(n)$-действия на грассманиане $\operatorname{Gr}(n, k)$. Тавтологическое векторное расслоение - это эрмитово векторное расслоение, связанное с фундаментальным представлением группы $U(k)$, которое обозначается $\mathcal{E}_{k}=S(n, k) \times_{U(k)} \mathbb{C}^{k}$, где $(s, v) \sim(s g, v g)$ и $s \in S(n, k), v \in \mathbb{C}^{k}, g \in U(k)$.

Легко проверить, что $i$-я строка матрицы $\sigma, \sigma_{i}: S(n, k) \rightarrow \mathbb{C}^{k}$ вида

$$
\sigma_{i}(s)=\left(z_{i}^{n-k+1}, z_{i}^{n-k+2}, \ldots, z_{i}^{n}\right), \quad s \in S(n, k), \quad i=1, \ldots, n,
$$

является $U(k)$-эквивариантной, т. е. она представляет собой (голоморфное) сечение расслоения $\mathcal{E}_{k}$. Более того, отображение $\mu_{(n, k)}=\sigma \sigma^{\dagger} \in M_{n}\left(C^{\infty}(\operatorname{Gr}(n, k))\right)$ - идемпотент ранга $k$. Оно представляет $\mathcal{E}_{k}$ в виде $\operatorname{Im} \mu_{(n, k)} \subset \mathbb{C}^{n}$. Наконец, справедливо равенство $\left(\mu_{(n, k)}\right)_{i j}=\left\langle\sigma_{i}, \sigma_{j}\right\rangle$, где $\langle z, w\rangle=\sum_{i=1}^{k} z_{i} \bar{w}_{i}$ означает обычное эрмитово произведение на $\mathbb{C}^{k}$.

3.2. Структура Пуассона на расслоении Штифеля. Рассмотрим стандартную структуру Пуассона-Ли на $U(n)$, полученную с помощью классической $r$-матрицы $r=1 / 2 \sum_{j<k} X_{j}^{k} \wedge Y_{j}^{k}$, где $X_{j}^{k}=e_{j}^{k}-e_{k}^{j}$ и $Y_{j}^{k}=i\left(e_{j}^{k}+e_{k}^{j}\right)$, а $e_{j}^{k}$ - матрица $n \times n$, в которой элемент $(j, k)$ равен единице, а все остальные элементы равны нулю. Напомним, что пуассонов бивектор определяется выражением $\pi=r^{L}-r^{R}$, в котором $L$ и $R$ означают левый и правый сдвиг. 
Можно показать, что $U(n-k)$ и $U(k)$ являются подгруппами Пуассона, поэтому однородные пространства

$$
S(n, k)=U(n) / U(n-k)
$$

и

$$
\operatorname{Gr}(n, k)=U(n) / U(n-k) \times U(k)
$$

наследуют структуру Пуассона. Более того, поскольку правые действия $U(k)$ и $U(n-k)$ коммутируют друг с другом, умножение справа на $U(k)$ сводится к правому пуассонову действию $U(k)$ на $S(n, k)$. Как следствие, главное расслоение (11) является пуассоновым, а связанное с ним расслоение $\mathcal{E}_{k}$ является пуассоновым векторным расслоением [6]. Эти утверждения следуют из плоской контрвариантной связности, явная формула для которой обсуждается в следующем разделе.

Дадим явное описание указанных пуассоновых структур в соответствии с симплектической редукцией, изложенной в предыдущем пункте.

Напомним, что для заданной биалгебры Ли $(\mathfrak{g}, \delta)$ и ее дуальной алгебры $\left(\mathfrak{g}^{*}, \delta^{*}\right)$ дубль Дринфельда алгебры $\mathfrak{g}$ является квазитриангулярной биалгебраической структурой $\mathcal{D}(\mathfrak{g})$, заданной на векторном пространстве $\mathfrak{g} \oplus \mathfrak{g}^{*}$ с $r$-матрицей, имеющей вид $r=\sum_{i} e^{i} \wedge e_{i}$, где $\left\{e_{i}\right\}-$ базис в $\mathfrak{g}$ и $\left\{e^{i}\right\}-$ дуальный базис в $\mathfrak{g}^{*}$. Биалгебры $(\mathfrak{g}, \delta)$ и $\left(\mathfrak{g}^{*}, \delta^{*}\right)$ являются биподалгебрами биалгебраической структуры $\mathcal{D}(\mathfrak{g})$. Тогда односвязная группа, интегрирующая $\mathcal{D}(\mathfrak{g})$, является группой Пуассона-Ли, таким образом $G$ и $G^{*}$ являются подгруппами Пуассона-Ли (см. книгу [11]).

Применим эту процедуру к стандартной структуре Пуассона--Ли группы $G=U(n)$. В этом случае дубль Дринфельда $G L(n, \mathbb{C})$ является действительной группой.

В работе [12] в явном виде описаны скобки структуры Пуассона на $S L(n, \mathbb{C})$, которые можно считать полуклассическим пределом для связей действительной квантовой группы $S L_{q}(n, \mathbb{C})$, описанной в статье [13]. Можно перенести данную структуру Пуассона непосредственно на $G L(n, \mathbb{C})$ и $\mathbb{C}^{n^{2}}$ согласно формулам

$$
\begin{gathered}
\left\{z_{i}^{j}, z_{i}^{j^{\prime}}\right\}=i z_{i}^{j} z_{i}^{j^{\prime}}, \quad j<j^{\prime}, \quad\left\{z_{i}^{j}, z_{i^{\prime}}^{j}\right\}=i z_{i}^{j} z_{i^{\prime}}^{j}, \quad i<i^{\prime}, \\
\left\{z_{i}^{j}, z_{i^{\prime}}^{j^{\prime}}\right\}=2 i z_{i}^{j^{\prime}} z_{i^{\prime}}^{j}, \quad i<i^{\prime}, \quad j<j^{\prime}, \quad\left\{z_{i}^{j}, z_{i^{\prime}}^{j^{\prime}}\right\}=0, \quad i>i^{\prime}, \quad j<j^{\prime}, \\
\left\{\bar{z}_{i}^{j}, z_{i}^{j}\right\}=2 i\left(\sum_{j^{\prime}>j}\left|z_{i}^{j^{\prime}}\right|^{2}-\sum_{i^{\prime}<i}\left|z_{i^{\prime}}^{j}\right|^{2}\right), \quad\left\{\bar{z}_{i}^{j}, z_{i^{\prime}}^{j^{\prime}}\right\}=0, \quad i \neq i^{\prime}, \quad j \neq j^{\prime}, \\
\left\{\bar{z}_{i}^{j}, z_{i^{\prime}}^{j}\right\}=i \bar{z}_{i}^{j} z_{i^{\prime}}^{j}+2 i \sum_{j^{\prime}>j} \bar{z}_{i}^{j^{\prime}} z_{i^{\prime}}^{j^{\prime}}, \quad i \neq i^{\prime}, \\
\left\{\bar{z}_{i}^{j}, z_{i}^{j^{\prime}}\right\}=-i \bar{z}_{i}^{j} z_{i}^{j^{\prime}}-2 i \sum_{i^{\prime}<i} \bar{z}_{i^{\prime}}^{j} z_{i^{\prime}}^{j^{\prime}}, \quad j \neq j^{\prime} .
\end{gathered}
$$

Заметим, что $(2,0)$-часть тензора Пуассона является голоморфным тензором Пуассона.

Посредством прямых вычислений можно доказать следующую лемму.

Лемма 3.1. 1. Отображение, заданное формулами (7), при $k=n$ определяет идеал $I_{c}=\left\langle\nu_{U(n)}(z)-c\right\rangle$ алгебры Пуассона на $C^{\infty}\left(\mathbb{C}^{n^{2}}\right)$ для любого числа $с \in \mathbb{R}$. В частности, $U(n)=\nu_{U(n)}^{-1}\left(1_{n}\right)$ является подмногообразием Пуассона. 
2. Подалгебра, порожденная матричными элементами последних $k$ столбцов матрицы $z=\left(z_{i}^{j}\right)$, является подалгеброй Пуассона, при этом проекиия из $\mathbb{C}^{n^{2}}$ в $\mathbb{C}^{n k}$, полученная путем игнорирования первых $n-k$ столбцов матрицы $z$, является пуассоновой.

3. Идеал $I_{c, k}=I_{c} \cap C^{\infty}\left(\mathbb{C}^{n k}\right)$ является идеалом Пуассона, порожденным идеалом $\left\langle\nu_{U(k)}(z)-c\right\rangle$. В частности, многообразие Штифеля $S(n, k)=\nu_{U(k)}^{-1}\left(1_{k}\right)$ является подмногообразием Пуассона пространства $\mathbb{C}^{n k}$.

Далее, пуассонову структуру многообразия Штифеля можно получить из скобок Пуассона (13), положив $j=n-k+1, \ldots, n$ и используя соотношения

$$
\sum_{i=1}^{n} \bar{z}_{i}^{j} z_{i}^{j^{\prime}}=\left(\sigma^{\dagger} \sigma\right)_{j}^{j^{\prime}}=\delta_{j}^{j^{\prime}}
$$

Эти скобки можно также получить как полуклассический предел для связей между генераторами алгебры квантового однородного пространства $S_{q}^{n, k}=U_{q}(n) / U_{q}(n-k)$ согласно работе [14].

Поскольку действие группы $U(k)$ на многообразии Штифеля является пуассоновым, получаем структуру Брюа-Пуассона $\pi_{\mathrm{bp}}$ на грассманиане $\operatorname{Gr}(n, k)=S(n, k) / U(k)$.

3.3. Структуры ПН на $\operatorname{Gr}(n, k)$ и $S(n, k)$. Рассмотрим геометрические концепции, обсуждаемые в предыдущем пункте. Пусть $\omega$ и $\pi$ - пресимплектическая форма и тензор Пуассона на $M$, при этом $\pi$ проектируема по отношению к пресимплектическому фактору $p: M \rightarrow \underline{M}$. Обозначим через $\underline{\omega}$ и $\underline{\pi}$ фактор симплектической формы и тензор Пуассона. Легко видеть, что $\omega \pi \omega=p^{*}(\underline{\omega} \underline{\pi} \underline{\omega})$.

Лемма 3.2. Триада $\left(\underline{M}, \underline{\omega}^{-1}, \underline{N}=\underline{\pi} \underline{\omega}\right)$ задает структуру ПН тогда и только тогда, когда $d \omega \pi \omega=0$.

ДокАЗАТЕЛьство. Поскольку $\underline{\omega}$ обратима, имеем $d \underline{\omega} \underline{\pi} \underline{\omega}=d b_{\underline{\omega}}(\pi)=b_{\underline{\omega}}\left(\left[\underline{\omega}^{-1}, \underline{\pi}\right]\right)$, где $b_{\underline{\omega}}: T M \rightarrow T^{*} M$ является сжатием под воздействием $\underline{\omega}$. Если $\left(\underline{N}, \underline{\omega}^{-1}\right)$ задает структуру ПН, то $\left[\underline{\omega}^{-1}, \underline{\pi}\right]=\left[\underline{\omega}^{-1}, \underline{N}(\underline{\pi})\right]=0$. Наоборот, если $\left[\underline{\omega}^{-1}, \underline{\pi}\right]=0$, тогда согласно результатам работы [9] пара $\left(\underline{N}, \underline{\omega}^{-1}\right)$ является структурой ПН. В этом случае справедливость утверждения является следствием инъективности $p^{*}$.

В книге [10] триада $(M, \omega, \pi)$, удовлетворяющая условию $d \omega \pi \omega=0$, называется $P \Omega$-структурой; в частности, $N=\pi \omega$ имеет нулевое кручение Нийенхейса. Рассмотрим теперь $M=S(n, k)$, где $\omega$ является формой $\Omega$, введенной в уравнении (6) и ограниченной на $S(n, k)$, а $\pi$ - тензор Пуассона, описанный в п. 3 леммы 3.1. Тогда верны равенства $\underline{\omega}=\Omega_{\mathrm{kks}}$ и $\underline{\pi}=\pi_{\mathrm{bp}}$.

ПреДЛОЖЕНИЕ 3.1. Форма $\Omega_{\mathrm{kks}}^{-1}$, обратная $\kappa$ симплектической форме $K К С$, и структура Брюа-Пуассона $\pi_{\mathrm{bp}}$ на грассманиане $\operatorname{Gr}(k, n)$ совместны друг с другом, т.е. $\left[\Omega_{\mathrm{kks}}^{-1}, \pi_{\mathrm{bp}}\right]=0$. Как следствие $\left(\operatorname{Gr}(k, n), \Omega_{\mathrm{kks}}^{-1}, \pi_{\mathrm{bp}} \Omega_{\mathrm{kks}}\right)$ является структурой $\Pi H$, а $(S(n, k), \Omega, \pi)$ является $P \Omega$-структурой.

ДокАзАтЕльство. Этот результат доказан в работе [5], но его можно наглядно представить следующим образом. Структура Брюа-Пуассона является фактором бивектора $\pi$ по $U(n)$, записанным в виде

$$
\pi=L_{g *} r-R_{g *} r, \quad r=i \sum_{1 \leqslant i<j \leqslant n} e_{i}^{j} \wedge e_{j}^{i},
$$


a $\Omega_{\mathrm{kks}}^{-1}-$ фактор формы

$$
\Omega^{-1}=-i L_{g *} \sum_{a \leqslant k, s>k} e_{a}^{s} \wedge e_{s}^{a}
$$

Вычислим скобку

$$
\left[\pi, \Omega^{-1}\right]=L_{g *}\left[\sum_{a<b \leqslant k} e_{a}^{b} \wedge e_{b}^{a}+\sum_{a \leqslant k<r} e_{a}^{r} \wedge e_{r}^{a}+\sum_{k<r<s} e_{r}^{s} \wedge e_{s}^{r}, \sum_{a \leqslant k<r} e_{a}^{r} \wedge e_{r}^{a}\right] .
$$

Второй член исчезает, первый член имеет следующий вид:

$$
\begin{aligned}
& {\left[\sum_{a<b \leqslant k} e_{a}^{b} \wedge e_{b}^{a}, \sum_{c \leqslant k<r} e_{c}^{r} \wedge e_{r}^{c}\right]=\sum_{a<b \leqslant k} \sum_{c \leqslant k<r} e_{a}^{b} \wedge e_{b}^{r} \wedge e_{r}^{c} \delta_{c}^{a}-e_{b}^{a} \wedge e_{a}^{r} \wedge e_{r}^{c} \delta_{c}^{b}+} \\
&+e_{a}^{b} \wedge e_{r}^{a} \wedge e_{c}^{r} \delta_{b}^{c}-e_{b}^{a} \wedge e_{r}^{b} \wedge e_{c}^{r} \delta_{a}^{c}=\sum_{c<b \leqslant k} e_{c}^{b} \wedge e_{b}^{r} \wedge e_{r}^{c}- \\
&-\sum_{a<c \leqslant k} e_{c}^{a} \wedge e_{a}^{r} \wedge e_{r}^{c}+\sum_{a<c \leqslant k} e_{a}^{c} \wedge e_{r}^{a} \wedge e_{c}^{r}-\sum_{c<b \leqslant k} e_{b}^{c} \wedge e_{r}^{b} \wedge e_{c}^{r}=0 .
\end{aligned}
$$

Третий член исчезает аналогичным образом. Утверждение, относящееся к $S(n, k)$, следует из леммы 3.2 .

\section{4. СПЕКТРАЛЬНАЯ ПРОБЛЕМА НА $\operatorname{Gr}(n, k)$}

Поскольку пуассоновы структуры Брюа и ККС совместны, они задают бигамильтонову систему [5]. Важным следствием этого факта является то, что существует пучок однородных пуассоновых структур $\pi_{t}=\pi_{\mathrm{bp}}+t \Omega_{\mathrm{kks}}^{-1}, t \in \mathbb{R}$, на грассманиане $\operatorname{Gr}(n, k)$. Более того, $(1,1)$-тензор $N=\pi_{\mathrm{bp}} \Omega_{\mathrm{kks}}: T \operatorname{Gr}(n, k) \rightarrow T \operatorname{Gr}(n, k)$ является тензором Нийенхейса, поэтому $\left(\operatorname{Gr}(n, k), \Omega_{\mathrm{kks}}^{-1}, N\right)$ является структурой ПН.

Докажем, что собственные числа тензора Нийенхейса являются переменными Гельфанда-Цетлина. Доказательство основано на использовании контрвариантной связности, заданной на пуассоновом векторном расслоении $\mathcal{E}_{k}$, введенном в п. 3.1.

Определение пуассонова векторного расслоения через скобку

$$
\{\cdot, \cdot\}_{\mathcal{E}}: C^{\infty}(M) \otimes \Gamma(\mathcal{E}) \rightarrow \Gamma(\mathcal{E}),
$$

где $\Gamma(\mathcal{E})$ обозначает гладкие сечения [15], можно также сформулировать с помощью плоской контрвариантной связности $\nabla: \Gamma(\mathcal{E}) \rightarrow \Gamma(T M \otimes \mathcal{E})$, заданной формулой

$$
\iota_{\mathrm{df}} \nabla(\sigma)=\{f, \sigma\}_{\mathcal{E}}, \quad f \in C^{\infty}(M), \quad \sigma \in \Gamma(\mathcal{E}) .
$$

Пусть $\nabla_{0}: \Gamma\left(\mathcal{E}_{k}\right) \rightarrow \Gamma\left(T \operatorname{Gr}(n, k) \otimes \mathcal{E}_{k}\right)$ - плоская контрвариантная связность расслоения $\mathcal{E}_{k}$, введенная в конце п. 3.1 , и пусть

$$
\nabla_{N}=\Omega_{\mathrm{kks}} \circ \nabla_{0}: \Gamma\left(\mathcal{E}_{k}\right) \rightarrow \Gamma\left(T^{*} \operatorname{Gr}(n, k) \otimes \mathcal{E}_{k}\right) \equiv \Omega^{1}\left(\mathcal{E}_{k}\right) .
$$

Далее, пусть $C_{ \pm}: \operatorname{Herm}(n) \rightarrow \mathfrak{b}(n, \mathbb{C})_{ \pm}$, где $\mathfrak{b}(n, \mathbb{C})_{ \pm}=\mathfrak{a} \oplus \mathfrak{n}_{ \pm}(\mathfrak{a}$ означает алгебру действительных диагональных матриц, а $\mathfrak{n}_{+}\left(\mathfrak{n}_{-}\right)$- строго верхняя (нижняя) диагональная комплексная матрица), имеет вид

$$
C_{+}(h)_{i j}=\left\{\begin{array}{ll}
2 h_{i j}, & i<j \\
h_{i i}, & i=j, \\
0, & i>j
\end{array} \quad h \in \operatorname{Herm}(n)\right.
$$

и $C_{-}=C_{+}^{\dagger}$. 
Следующее предложение можно доказать с помощью явных формул (13).

ПрЕДЛОЖЕНИЕ 4.1. Для Каждого сечения $\sigma_{i}$, заданного выражением (12), справедливо равенство

$$
\nabla_{N}\left(\sigma_{i}\right)=\sum_{r} d\left(C_{-}\left(\mu_{(n, k)}\right)_{i r}\right) \sigma_{r} \in \Omega^{1}\left(\mathcal{E}_{k}\right)
$$

где $C_{-}$- отображение, введенное по формуле (14).

ДокАзАтЕльство. Бивектор $\pi$ на $U(n)$ можно записать в виде

$$
\pi=i\left(\sum_{i, j ; r<s} z_{i}^{s} \frac{\partial}{\partial z_{i}^{r}} \wedge z_{j}^{r} \frac{\partial}{\partial z_{j}^{s}}-\sum_{i, j ; r<s} z_{r}^{i} \frac{\partial}{\partial z_{s}^{i}} \wedge z_{s}^{j} \frac{\partial}{\partial z_{r}^{j}}\right) .
$$

Несмотря на то что эта пуассонова структура выглядит голоморфной, у нее есть смешанные компоненты из-за того, что в $U(n)$ справедливо равенство $\sum_{k} z_{i}^{k} \bar{z}_{j}^{k}=\delta_{i}^{j}$, поэтому $\partial_{z} \bar{z} \neq 0$. Здесь мы используем соотношение $d \bar{z}_{j}^{i}=-\sum_{k, l} \bar{z}_{k}^{i} d z_{k}^{l} \bar{z}_{j}^{l}$. Нам нужно вычислить $N^{*} d \sigma_{i}=\Omega \pi^{\#} d z_{i}^{j}$ при $j=n-k+1, \ldots, n$ и $i=1, \ldots, n$. Прямые расчеты приводят к следующему результату:

$$
\pi^{\#} d z_{i}^{j}=-i \sum_{l, r}(-1)^{r>j} z_{i}^{r} z_{l}^{j} \frac{\partial}{\partial z_{l}^{r}}-i \sum_{l, r}(-1)^{r>i} z_{i}^{l} z_{r}^{j} \frac{\partial}{\partial z_{r}^{l}},
$$

где

$$
(-1)^{r>j}=\left\{\begin{array}{rr}
1, & r>j \\
-1, & r<j \\
0, & r=j
\end{array}\right.
$$

Таким образом, получаем равенство

$$
N^{*} d z_{i}^{j}=-\sum_{l ; r \leqslant n-k} z_{i}^{r} z_{l}^{j} d \bar{z}_{l}^{r}-\sum_{r}(-1)^{r>i} d \mu_{i r} z_{r}^{j},
$$

где $\mu_{i j}=\mu_{(n, k)_{i j}}=\sum_{s>n-k} z_{i}^{s} \bar{z}_{j}^{s}$ - отображение моментов $U(n)$-действия. Перепишем второй член в виде

$$
\sum_{r}(-1)^{r>i} d \mu_{i r} z_{r}^{j}=-2 \sum_{r<i} d \mu_{i r} z_{r}^{j}+\sum_{l ; s \leqslant n-k} z_{i}^{s} \bar{z}_{l}^{s} d z_{l}^{j}-d \mu_{i i} z_{i}^{j} .
$$

Подводя итог, получаем, что

$$
N^{*} d \sigma_{i}=d \mu_{i i} \sigma_{i}+2 \sum_{r<i} d \mu_{i r} \sigma_{r}=\sum_{r} d\left(C_{-}(\mu)_{i r}\right) \sigma_{r} .
$$

Напомним, что переменные Гельфанда-Цетлина суть собственные числа $\lambda_{\alpha}^{(r)}$ верхнего левого минора $\mu_{(n, k)}^{(r)}$ порядка $r$ оператора $\mu_{(n, k)}$. Обозначим через $C_{-}\left(d \mu_{(n, k)}\right)^{(r)}$ верхний левый минор порядка $r$ оператора $C_{-}\left(d \mu_{(n, k)}\right)$. Следующая лемма является прямым следствием предложения 4.1. 
ЛЕммА 4.1. Каждый минор $\mu_{(n, k)}^{(r)}, r=1, \ldots, n$, удовлетворяет уравнению

$$
N^{*} d \mu_{(n, k)}^{(r)}=C_{-}\left(d \mu_{(n, k)}\right)^{(r)} \mu_{(n, k)}^{(r)}+\mu_{(n, k)}^{(r)} C_{-}\left(d \mu_{(n, k)}\right)^{(r) \dagger} .
$$

ДокАЗАТЕЛЬство. Доказательство проводится прямым вычислением согласно предложению 4.1.

Уравнение (15) в работе [6] называется мастер-уравнением и является ключевым уравнением, которое надо решить при диагонализации тензора Нийенхейса для эрмитовых симметричных пространств. В статье [6] показано, что собственные числа тензора Нийенхейса определяются отличными от констант собственными числами решения мастер-уравнения. Мы предлагаем другое доказательство этого факта.

ПреДЛОжЕНИЕ 4.2. Для любой переменной Гельфанда-Цетлина $\lambda_{\alpha}^{(r)}$ число $2 \lambda_{\alpha}^{(r)}$ является собственным числом тензора Нийенхейса $N^{*}=\Omega_{\mathrm{kks}} \pi_{\mathrm{bp}}^{\#}$.

ДоказАтеЛЬСтво. Обозначим как $v_{\alpha}^{(r)} \in \mathbb{C}^{r}$ нормированный собственный вектор, соответствующий $\lambda_{\alpha}^{(r)}$. Легко проверить, что для плотного открытого подмножества, в котором собственные числа оператора $\mu_{(n, k)}^{(r)}$ различны, справедливы равенства

$$
\lambda_{\alpha}^{(r)}=\left(v_{\alpha}^{(r)}, \mu_{(n, k)}^{(r)} v_{\alpha}^{(r)}\right), \quad d \lambda_{\alpha}^{(r)}=\left(v_{\alpha}^{(r)}, d \mu_{(n, k)}^{(r)} v_{\alpha}^{(r)}\right),
$$

где $(\cdot, \cdot)$ - эрмитово внутреннее произведение. Таким образом, мы видим, что

$$
\begin{aligned}
N^{*} d \lambda_{\alpha}^{(r)}= & \left(v_{\alpha}^{(r)}, N^{*} d \mu_{(n, k)}^{(r)} v_{\alpha}^{(r)}\right)=\left(v_{\alpha}^{(r)},\left(C_{-}\left(d \mu_{(n, k)}\right)^{(r)} \mu_{(n, k)}^{(r)}+\right.\right. \\
& \left.\left.+\mu_{(n, k)}^{(r)} C_{-}\left(d \mu_{(n, k)}\right)^{(r) \dagger}\right) v_{\alpha}^{(r)}\right)=\lambda_{\alpha}^{(r)}\left(v_{\alpha}^{(r)},\left(C_{-}\left(d \mu_{(n, k)}\right)^{(r)}+\right.\right. \\
& \left.\left.+C_{-}\left(d \mu_{(n, k)}\right)^{(r) \dagger}\right) v_{\alpha}^{(r)}\right)=2 \lambda_{\alpha}^{(r)}\left(v_{\alpha}^{(r)}, d \mu_{(n, k)}^{(r)} v_{\alpha}^{(r)}\right)=2 \lambda_{\alpha}^{(r)} d \lambda_{\alpha}^{(r)} .
\end{aligned}
$$

Поскольку переменные Гельфанда-Цетлина независимы, то структура ПН на грассманиане $\operatorname{Gr}(n, k)$ имеет максимальный ранг. Как следствие существует интегрируемая модель на симплектическом группоиде, интегрирующая структуру Брюа-Пуассона. Остается нерешенной проблема объяснения поведения поверхностей уровней собственных чисел $\lambda_{\alpha}^{(r)}$ с кратностью больше одного. Мы надеемся, что явный подход, описанный в данной работе, поможет прояснить этот вопрос.

\section{Список литературы}

[1] E. Hawkins, J. Symplectic Geom., 6:1 (2008), 61-125.

[2] F. Bonechi, N. Ciccoli, N. Staffolani, M. Tarlini, J. Geom. Phys., 62:8 (2012), 1851-1865.

[3] F. Bonechi, N. Ciccoli, J. Qiu, M. Tarlini, Commun. Math. Phys., 331:2 (2014), 851-885.

[4] F. Bonechi, Multiplicative integrable models from Poisson-Nijenhuis structures, arXiv: 1507.01500.

[5] S. Khoroshkin, A. Radul, V. Rubtsov, Commun. Math. Phys., 152:2 (1993), 299-315.

[6] F. Bonechi, J. Qiu, M. Tarlini, Complete integrability from Poisson-Nijenhuis structures on compact hermitian symmetric spaces, arXiv: 1503.07339.

[7] T. Nishinou, Y. Nohara, K. Ueda, Adv. Math., 224:2 (2010), 648-706.

[8] V. Guillemin, S. Sternberg, J. Funct. Anal., 52:1 (1983), 106-128.

[9] I. Vaisman, Rend. Sem. Mat. Univ. Politec. Torino, 52:4 (1994), 377-394. 
[10] F. Magri, C. Morosi, A Geometrical Characterization of Hamiltonian Systems Through the Theory of Poisson-Nijenhuis Manifolds, University of Milan, Milan, 1984.

[11] V. Chari, A. Pressley, Quantum Groups, Cambridge Univ. Press, Cambridge, 1994.

[12] S. Zakrzewski, J. Phys. A: Math. Gen., 30:18 (1997), 6535-6543.

[13] P. Podlés, Publ. Res. Inst. Math. Sci., 28:5 (1992), 709-745.

[14] G. B. Podkolzin, L. I. Vainerman, Pacific J. Math., 188:1 (1999), 179-199.

[15] V. L. Ginzburg, J. Symplectic Geom., 1:1 (2001), 121-170. 